Claremont Colleges

Scholarship@ Claremont

All HMC Faculty Publications and Research

HMC Faculty Scholarship

5-19-2015

\title{
Direct, biomimetic synthesis of $(+)$-artemone via a stereoselective, organocatalytic cyclization
}

Eric D. Nacsa

Columbia University

Brian C. Fielder

Shannon P. Wetzler

Harvey Mudd College

Veerasak Srisuknimit

Harvard University

Jonathan P. Litz

University of Washington

See next page for additional authors

\section{Recommended Citation}

Direct, biomimetic synthesis of (+)-artemone via a stereoselective, organocatalytic cyclization,” Nacsa, E.D.; Fielder, B.C.; Wetzler, S.P.; Srisuknimit, V.; Litz, J.P.; Van Vleet, M.J.; Quach, K.; Vosburg, D.A. Synthesis 2015

This Article is brought to you for free and open access by the HMC Faculty Scholarship at Scholarship @ Claremont. It has been accepted for inclusion in All HMC Faculty Publications and Research by an authorized administrator of Scholarship @ Claremont. For more information, please contact scholarship@cuc.claremont.edu. 
Authors

Eric D. Nacsa, Brian C. Fielder, Shannon P. Wetzler, Veerasak Srisuknimit, Jonathan P. Litz, Mary J. Van Vleet, Kim Quach, and David A. Vosburg 


\section{Direct, Biomimetic Synthesis of (+)-Artemone via a Stereoselective, Organocatalytic Cyclization}

\author{
Eric D. Nacsa \\ Brian C. Fielder \\ Shannon P. Wetzler \\ Veerasak Srisuknimit \\ Jonathan P. Litz \\ Mary J. Van Vleet \\ Kim Quach \\ David A. Vosburg*
}

Department of Chemistry, Harvey Mudd College, 301 Platt Boulevard, Claremont, CA 91711, USA vosburg@hmc.edu

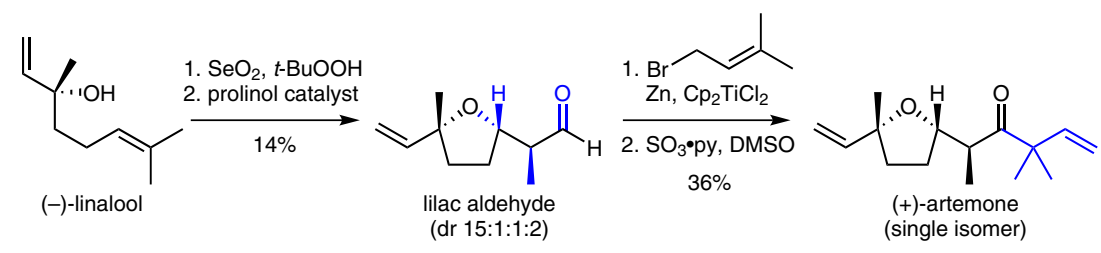

Received: 07.03.2015

Accepted after revision: 07.04.2015

Published online: 19.05 .2015

DOI: 10.1055/s-0034-1380684; Art ID: ss-2015-m0145-op

Abstract We present a four-step synthesis of (+)-artemone from (-)linalool, featuring iminium organocatalysis of a doubly diastereoselective conjugate addition reaction. The strategy follows a proposed biosynthetic pathway, rapidly generates stereochemical complexity, uses no protecting groups, and minimizes redox manipulations.

Key words natural products, cyclization, terpenoids, total synthesis, stereoselective synthesis, green chemistry, organocatalysis, biomimetic synthesis

Artemone is a sesquiterpene natural product from the Indian sage Artemisia pallens. ${ }^{1}$ Some of the other metabolites from this plant have been shown to possess useful medicinal properties, ${ }^{2}$ though none have yet been disclosed for artemone. Many related compounds have desirable olfactory properties. ${ }^{3}$

The only reported stereoselective syntheses of artemone are a 20 -step route by Honda and co-workers, ${ }^{4}$ which results in a 1:2 mixture of artemone and davanone, and our recent six-step synthesis via a diastereoselective allylic 0alkylation. $^{5}$ We sought a shorter route to artemone and looked for inspiration to the plausible biosynthetic pathway in Scheme $1,^{6}$ an alternative biomimetic approach to our previous studies on davanone. ${ }^{7}$ We propose that the final steps in the biosynthesis of artemone are an allylic oxidation and an intramolecular conjugate addition reaction. The latter step generates the ring and introduces two new stereocenters in a single operation.

Our strategy is similar to that of Naegeli and co-workers, though their early work lacked stereocontrol, resulting in an equal mixture of all eight possible stereoisomers. ${ }^{1 \mathrm{a}, 8}$ Thus, a key element to our synthesis is the identification of

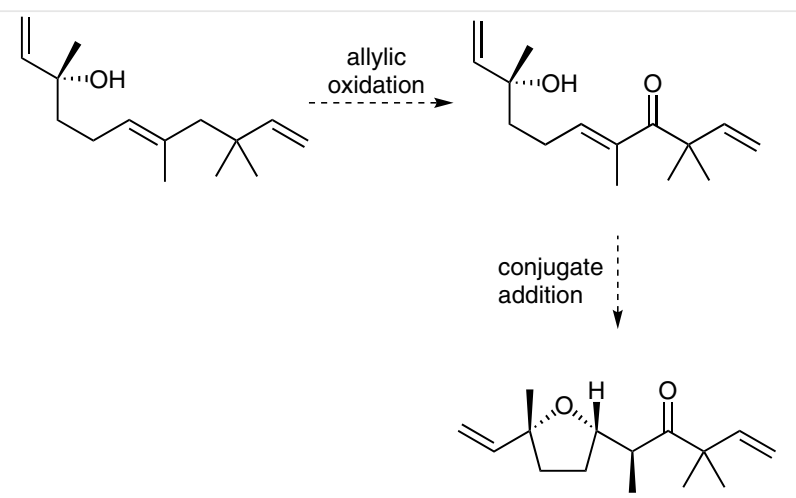

(+)-artemone

Scheme 1 Proposed biosynthesis of artemone

a stereoselective conjugate addition reaction. We were also guided by Gaich and Baran's description of an ideal synthesis $^{9}$ and aimed to avoid protecting groups and unnecessary functional group transformations, even being willing to sacrifice chemical yield for a maximally direct route.

We selected (-)-linalool, an inexpensive monoterpene, as our starting material (Scheme 2). The selenium dioxide mediated oxidation of linalool to enal $\mathbf{1}$ is a known reaction. ${ }^{10}$ While we never obtained the impressively high yield (79\%) that Sharma and Chand reported for this transformation, we could reliably access $\mathbf{1}$ at room temperature or with microwave heating. Column chromatography readily separated the enal from residual linalool and the intermediate diol.

The conjugate addition step is pivotal, as it establishes the remaining two stereocenters not already present in linalool. This is a challenging reaction, however, as the tertiary alcohol is a poor nucleophile and the $\alpha$-methyl group disfavors enal activation with bulky amines or metal-ligand complexes. Also, the vast majority of published stereoselec- 
<smiles>C=CC(C)(O)CCC=C(C)C</smiles>

$(-)$-linalool
$52 \%$

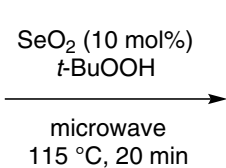

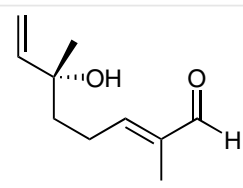
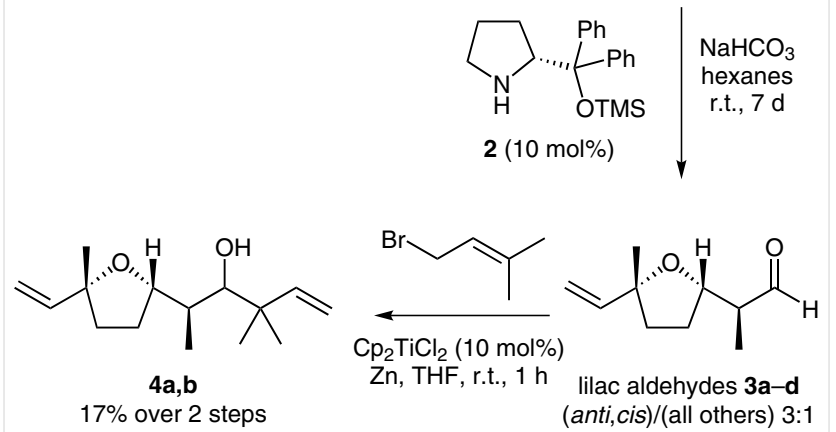

$$
\begin{array}{l|c}
\mathrm{SO}_{3} \bullet p y & \\
\mathrm{DMSO}^{2} & \text { r.t., } 2 \mathrm{~d} \\
\mathrm{CH}_{2} \mathrm{Cl}_{2} & 57 \%
\end{array}
$$

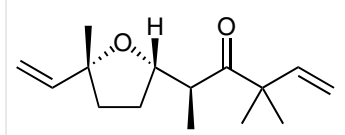

(+)-artemone (5)

Scheme 2 Four-step synthesis of (+)-artemone

tive conjugate addition reactions merely offer stereoselectivity at the $\beta$-position and not at both the $\beta$ - and $\alpha$-carbons. ${ }^{11}$ Our transformation requires stereoselectivity in the nucleophilic attack as well as the subsequent $\alpha$-protonation step.

After screening a number of organic and inorganic catalysts known to activate enals, we established that Jørgensen-Hayashi catalyst $\mathbf{2}^{12}$ (Scheme 2) conferred the greatest selectivity in this reaction. Other optimal parameters included a nonpolar solvent such as hexanes or cyclohexane, performing the reaction at room temperature, and the addition of sodium bicarbonate. Our most selective conditions provided diastereomeric ratios of up to $5: 1$ for the desired anti,cis-lilac aldehyde (3) versus the sum of the other three (anti,trans, syn,trans, and syn,cis) lilac aldehydes that are also products of this reaction; however, this level of selectivity was only observed early in the reaction. To obtain useful amounts of product, prolonged exposure to the catalyst was necessary and resulted in degraded stereoselectivity. We found the most practical balance of selectivity and yield required one week at room temperature. These conditions were $65-75 \%$ stereoselective for the desired anti,cis-lilac aldehyde (3a). Column chromatography removed unconverted enal $\mathbf{1}$ and residual catalyst $\mathbf{2}$, but lilac aldehydes 3a-d were inseparable from each other; further purification was achieved after the subsequent prenylation step.

Addition of the $\gamma$-prenyl group was most convenient using Fleury and Ashfeld's titanium-catalyzed organozinc addition protocol. ${ }^{13}$ Despite the fact that this reaction introduced an additional stereocenter, the desired epimeric secondary alcohols $\mathbf{4 a , b}$ could be isolated more readily by column chromatography than their lilac aldehyde precursor (3a). The sterically congested alcohols $\mathbf{4 a}$ and $\mathbf{4 b}$ were oxidized slowly but cleanly to (+)-artemone (5), completing the synthesis. Our synthetic artemone matched the data of Honda and co-workers ${ }^{4}$ and material obtained from our previous route. ${ }^{5}$

In conclusion, we have described a very short, biomimetic synthesis of (+)-artemone from (-)-linalool using an organocatalytic cyclization. This route avoids protecting groups and is redox economic. ${ }^{14}$ The two-step preparation of lilac aldehyde (3) is much more direct and atom economic than Sabitha and co-workers' eight-step synthesis, ${ }^{15}$ though our yields and selectivities are more modest.

All reactions, unless otherwise noted, were carried out under an atmosphere of argon using oven-dried glassware and magnetic stirring. Anhydrous hexanes, THF, and $\mathrm{CH}_{2} \mathrm{Cl}_{2}$ were obtained from a SolvPure solvent system using activated alumina. Microwave reactions were performed with a Biotage Initiator 2.5 system. Reactions were monitored by TLC on glass plates coated with 400-mesh silica gel and visualized using UV radiation and either anisaldehyde or ceric sulfate stain. Column chromatography was performed using 60-mesh silica gel. IR spectra were recorded on a Thermo Scientific Nicolet iS5 FT-IR spectrometer using an iD5 diamond ATR accessory. GC-MS was performed with an Agilent 5975C system using electron-impact ionization. ${ }^{1} \mathrm{H}$ and ${ }^{13} \mathrm{C}$ NMR spectra were obtained on a Bruker Avance 400 spectrometer at ambient temperature. Chemical shifts are expressed in ppm $(\delta)$ using TMS as the internal standard. For ${ }^{13} \mathrm{C}$ spectra, chemical shifts are referenced to $\mathrm{CDCl}_{3}$ at $77.0 \mathrm{ppm}$. Optical rotation measurements were made using a Jasco P-1010 polarimeter. High-resolution mass spectrometry (HRMS) was performed using a chemical ionization (CI) source on an Agilent 6210 time-of-flight mass spectrometer.

\section{(3R,6E)-8-0xolinalool (1)}

Depending on the time available and scale required, hydroxyenal $\mathbf{1}$ was prepared by either a conventional or a microwave procedure.

Conventional Procedure: (-)-Linalool (7.13 g, $46 \mathrm{mmol}$ ) was dissolved in $\mathrm{CH}_{2} \mathrm{Cl}_{2}(10 \mathrm{~mL})$. Separately, $\mathrm{SeO}_{2}(554.8 \mathrm{mg}, 5.00 \mathrm{mmol})$ and $70 \%$ aq $t$-BuOOH $(41.0 \mathrm{~mL}, 38.6 \mathrm{~g}, 300 \mathrm{mmol})$ were added to $\mathrm{CH}_{2} \mathrm{Cl}_{2}(85 \mathrm{~mL})$ in a round-bottom flask. To the resulting cloudy mixture, the (-)-linalool solution was added. The reaction mixture was stirred at r.t. for 7 $\mathrm{d}$, and then diluted with additional $\mathrm{CH}_{2} \mathrm{Cl}_{2}(105 \mathrm{~mL})$. The organic layer was washed successively with $2.0 \mathrm{M} \mathrm{KOH}(105 \mathrm{~mL})$, water $(105 \mathrm{~mL})$, and brine $(105 \mathrm{~mL})$, then dried $\left(\mathrm{MgSO}_{4}\right)$, filtered, and concentrated. The crude product was purified using flash chromatography (hexanes-EtOAc, 5:1) to afford hydroxyenal $\mathbf{1}$ as a slightly yellow oil; yield: $1.22 \mathrm{~g}(16 \%)$. 
Microwave Procedure: To a microwave vial were added $\mathrm{SeO}_{2}(53.3 \mathrm{mg}$, $0.48 \mathrm{mmol}$ ) and $70 \%$ aq $t$-BuOOH (3.96 mL, $3.7 \mathrm{~g}, 28.8 \mathrm{mmol})$, followed by a solution of (-)-linalool $(0.73 \mathrm{~g}, 4.8 \mathrm{mmol})$ in anhydrous $\mathrm{CH}_{2} \mathrm{Cl}_{2}(9 \mathrm{~mL})$. The vial was sealed and the reaction mixture was stirred under microwave irradiation $(250 \mathrm{~W})$ at $115^{\circ} \mathrm{C}$ for $20 \mathrm{~min}$. The two-layer reaction mixture was diluted with $\mathrm{CH}_{2} \mathrm{Cl}_{2}(20 \mathrm{~mL})$ and washed with $2.0 \mathrm{M} \mathrm{KOH}(20 \mathrm{~mL})$, deionized water $(20 \mathrm{~mL})$, and brine $(20 \mathrm{~mL})$. The organic layer was dried $\left(\mathrm{MgSO}_{4}\right)$, filtered, and concentrated. The crude product was purified using flash chromatography (hexanes-EtOAc, 5:1) to afford hydroxyenal $\mathbf{1}$ as a slightly yellow oil; yield: $0.42 \mathrm{~g}(52 \%)$.

$[\alpha]_{D}^{23}-12.0\left(\right.$ c 1.0, $\left.\mathrm{CHCl}_{3}\right) ; R_{f}=0.56$ (hexanes-EtOAc, $4: 1$ ).

IR: $3458,1739,1685,1640 \mathrm{~cm}^{-1}$.

${ }^{1} \mathrm{H}$ NMR $\left(400 \mathrm{MHz}, \mathrm{CDCl}_{3}\right): \delta=9.39(\mathrm{~s}, 1 \mathrm{H}), 6.50(\mathrm{dt}, J=1.3,7.4 \mathrm{~Hz}, 1$ H), $5.93(\mathrm{dd}, J=11,17 \mathrm{~Hz}, 1 \mathrm{H}), 5.26(\mathrm{dd}, J=1.0,17 \mathrm{~Hz}, 1 \mathrm{H}), 5.13(\mathrm{dd}$, $J=1.0,11 \mathrm{~Hz}, 1 \mathrm{H}), 2.37-2.44(\mathrm{~m}, 2 \mathrm{H}), 1.66-1.76$ (m, $2 \mathrm{H}), 1.74$ (s, 3 $\mathrm{H}), 1.60$ (br s, $1 \mathrm{H}), 1.34(\mathrm{~s}, 3 \mathrm{H})$.

${ }^{13} \mathrm{C}$ NMR $\left(100 \mathrm{MHz}, \mathrm{CDCl}_{3}\right): \delta=195.3,155.1,144.2,138.9,112.2,72.6$, 40.1, 27.9, 23.7, 8.9.

HRMS: $m / z\left[\mathrm{M}+\mathrm{H}^{+}\right]$calcd for $\mathrm{C}_{10} \mathrm{H}_{17} \mathrm{O}_{2}$ : 169.1223; found: 169.1225 .

\section{2-(5-Methyl-5-vinyltetrahydrofuran-2-yl)propionaldehyde (Lilac Aldehydes, 3a-d)}

To hydroxyenal 1 (201.5 mg, $1.20 \mathrm{mmol}$ ) were added hexanes (10 $\mathrm{mL})$, organocatalyst $2(5.5 \mathrm{mg} / \mathrm{mL}$ in hexanes, $7.04 \mathrm{~mL}, 0.12 \mathrm{mmol})$, and $\mathrm{NaHCO}_{3}(303.5 \mathrm{mg}, 3.61 \mathrm{mmol})$. The reaction mixture was stirred at r.t. for $7 \mathrm{~d}$, then was diluted with hexanes $(20 \mathrm{~mL})$. The solution was washed with $1 \mathrm{M} \mathrm{HCl}(2 \times 20 \mathrm{~mL})$, sat. aq $\mathrm{NaHCO}_{3}(20 \mathrm{~mL})$, and brine $(20 \mathrm{~mL})$. The organic layer was dried $\left(\mathrm{MgSO}_{4}\right)$, concentrated, and purified using flash chromatography (hexanes-EtOAc, 20:1) to provide an anti,cis-enriched mixture of lilac aldehydes (3a-d); yield: $54.3 \mathrm{mg}$ (27\%). Although chromatography did not separate 3a from its diastereomers, it did remove residual traces of organocatalyst $\mathbf{2}$ which otherwise promoted undesired diastereomeric equilibration to give equal amounts of 3a-d. The diastereomeric mixture (typically containing $65-75 \%$ 3a) was carried on through the next step.

$R_{f}=0.49$ (hexanes-EtOAc, 5:1).

IR: $1727 \mathrm{~cm}^{-1}$.

${ }^{1} \mathrm{H}$ NMR (400 MHz, $\mathrm{CDCl}_{3}$ ): $\delta$ (characteristic peaks) $=9.81(\mathrm{~d}, J=2.5$ $\mathrm{Hz}, 1 \mathrm{H}, 3 \mathbf{a}-\mathrm{CHO}), 9.80$ (d, $J=1.3 \mathrm{~Hz}, 1 \mathrm{H}, \mathbf{3 b}-\mathrm{CHO}), 9.79(\mathrm{~d}, J=2.3 \mathrm{~Hz}$, $1 \mathrm{H}, 3 \mathbf{c}-\mathrm{CHO}), 9.77$ (d, $J=1.5 \mathrm{~Hz}, 1 \mathrm{H}, 3 \mathrm{~d}-\mathrm{CHO}), 1.16(\mathrm{~d}, J=7.0 \mathrm{~Hz}, 3$ $\left.\mathrm{H}, 3 \mathbf{d} \mathrm{CHCH}_{3}\right), 1.12$ (d, $\left.J=7.0 \mathrm{~Hz}, 3 \mathrm{H}, 3 \mathbf{b} \mathrm{CHCH}_{3}\right), 1.08(\mathrm{~d}, J=7.0 \mathrm{~Hz}, 3$ $\left.\mathrm{H}, 3 \mathbf{c} \mathrm{CHCH}_{3}\right), 1.05$ (d, $J=7.0 \mathrm{~Hz}, 3 \mathrm{H}, 3 \mathbf{a} \mathrm{CHCH}_{3}$ ).

HRMS: $m / z\left[\mathrm{M}+\mathrm{H}^{+}\right]$calcd for $\mathrm{C}_{10} \mathrm{H}_{17} \mathrm{O}_{2}$ : 169.1223; found: 169.1229 .

\section{4,4-Dimethyl-2-(5-methyl-5-vinyltetrahydrofuran-2-yl)hex-5-en- 3-ol (Artemols, 4a,b)}

Zinc dust (56.3 mg, $0.861 \mathrm{mmol}$ ) and $\mathrm{Cp}_{2} \mathrm{TiCl}_{2}(8.2 \mathrm{mg}, 0.033 \mathrm{mmol})$ were added to THF ( $5 \mathrm{~mL}$ ). The flask was sealed under nitrogen. Upon stirring for $10 \mathrm{~min}$, the reaction color progressed from blood red to green. In a separate vial, the above mixture of 3a-d $(54.0 \mathrm{mg}, 0.321$ $\mathrm{mmol})$ and 3,3-dimethylallyl bromide $(0.11 \mathrm{~mL}, 143.5 \mathrm{mg}, 0.963$ $\mathrm{mmol}$ ) were dissolved in THF (1 mL). This solution was then added to the reaction mixture. After $1 \mathrm{~h}$, sat. aq $\mathrm{NH}_{4} \mathrm{Cl}(10 \mathrm{~mL})$ and $\mathrm{Et}_{2} \mathrm{O}(10 \mathrm{~mL})$ were added. The reaction mixture was extracted with $\mathrm{Et}_{2} \mathrm{O}(3 \times 10$ $\mathrm{mL})$. The organic layers were combined, washed with brine $(30 \mathrm{~mL})$, dried $\left(\mathrm{MgSO}_{4}\right)$, and concentrated. The residue was then purified using flash chromatography (hexanes-EtOAc, 50:1 $\rightarrow$ 25:1) to afford 28.1 $\mathrm{mg}$ of artemol $\mathbf{4 a}$ and $20.9 \mathrm{mg}$ of artemol $\mathbf{4 b}$ (17\% combined two-step yield from $\mathbf{1}$ ) as clear, colorless oils.

\section{Alcohol 4a}

$[\alpha]_{D}^{23}+9.2\left(c\right.$ 1.7, $\left.\mathrm{CHCl}_{3}\right) ; R_{f}=0.41$ (hexanes-EtOAc, 5:1).

IR: $3466 \mathrm{~cm}^{-1}$.

${ }^{1} \mathrm{H}$ NMR $\left(400 \mathrm{MHz}, \mathrm{CDCl}_{3}\right): \delta=5.98(\mathrm{~m}, 1 \mathrm{H}), 5.91(\mathrm{dd}, J=11,17 \mathrm{~Hz}, 1$ $\mathrm{H}), 5.18$ (dd, $J=1.6,17 \mathrm{~Hz}, 1 \mathrm{H}), 5.04(\mathrm{app} \mathrm{s}, 1 \mathrm{H}), 5.00$ (dd, $J=1.5,6.4$ $\mathrm{Hz}, 1 \mathrm{H}), 4.97(\mathrm{dd}, J=1.6,11 \mathrm{~Hz}, 1 \mathrm{H}), 3.93(\mathrm{~m}, 1 \mathrm{H}), 3.73(\mathrm{~d}, J=3.9 \mathrm{~Hz}$, $1 \mathrm{H}), 1.57-1.98$ (m, $5 \mathrm{H}), 1.29$ (s, $3 \mathrm{H}), 1.08$ (s, $3 \mathrm{H}), 1.06$ (s, $3 \mathrm{H}), 0.88$ (d, $J=7.0 \mathrm{~Hz}, 3 \mathrm{H})$.

${ }^{13} \mathrm{C}$ NMR $\left(100 \mathrm{MHz}, \mathrm{CDCl}_{3}\right): \delta=146.0,144.5,112.1,111.5,82.8,82.5$, 76.7, 41.9, 38.9, 38.0, 29.7, 26.4, 24.8, 23.9, 11.3.

HRMS: $m / z\left[\mathrm{M}+\mathrm{H}^{+}\right]$calcd for $\mathrm{C}_{15} \mathrm{H}_{27} \mathrm{O}_{2}$ : 239.2006; found: 239.2001 .

\section{Alcohol 4b}

$R_{f}=0.63$ (hexanes-EtOAc, 5:1).

${ }^{1} \mathrm{H}$ NMR (400 MHz, $\mathrm{CDCl}_{3}$ ): $\delta=5.94(\mathrm{dd}, J=11,17 \mathrm{~Hz}, 1 \mathrm{H}$ ), 5.87 (dd, $J=11,17 \mathrm{~Hz}, 1 \mathrm{H}), 5.20(\mathrm{dd}, J=1.6,17 \mathrm{~Hz}, 1 \mathrm{H}), 5.01(\mathrm{dd}, J=1.6,11 \mathrm{~Hz}$, $1 \mathrm{H}), 4.95-4.98(\mathrm{~m}, 2 \mathrm{H}), 4.34(\mathrm{~d}, J=6.8 \mathrm{~Hz}, 1 \mathrm{H}), 3.97$ (ddd, $J=5.1,9.7$, $9.7 \mathrm{~Hz}, 1 \mathrm{H}), 3.22$ (app t, $J=6.8 \mathrm{~Hz}, 1 \mathrm{H}), 1.99(\mathrm{~m}, 1 \mathrm{H}), 1.89(\mathrm{~m}, 1 \mathrm{H})$, 1.67-1.76 (m, $2 \mathrm{H}), 1.48-1.57(\mathrm{~m}, 1 \mathrm{H}), 1.30(\mathrm{~s}, 3 \mathrm{H}), 1.06(\mathrm{~s}, 3 \mathrm{H}), 1.05$ $(\mathrm{s}, 3 \mathrm{H}), 0.86(\mathrm{~d}, J=6.9 \mathrm{~Hz}, 3 \mathrm{H})$.

${ }^{13} \mathrm{C}$ NMR $\left(100 \mathrm{MHz}, \mathrm{CDCl}_{3}\right): \delta=146.6,144.2,111.5,111.2,83.5,83.1$, $77.2,42.7,41.0,37.3,31.3,27.0,25.2,21.5,17.6$.

(2S)-4,4-Dimethyl-2-[(2S,5R)-5-methyl-5-vinyltetrahydrofuran-2yl]hex-5-en-3-one (anti,cis-Artemone, 5)

A solution of artemol (4a; $26.9 \mathrm{mg}, 0.113 \mathrm{mmol})$ in $\mathrm{CH}_{2} \mathrm{Cl}_{2}-\mathrm{DMSO}$ $(4: 1,1 \mathrm{~mL})$ was stirred for $2 \mathrm{~min}$ at r.t., followed by $7 \mathrm{~min}$ at $0{ }^{\circ} \mathrm{C}$. $\mathrm{Et}_{3} \mathrm{~N}$ $(0.14 \mathrm{~mL}, 1.03 \mathrm{mmol})$ and sulfur trioxide-pyridine complex (119 mg, $0.749 \mathrm{mmol}$ ) were added and the reaction mixture was allowed to warm to r.t. The solution changed from pale yellow to deep red. After $2 \mathrm{~d}$, the reaction was quenched with sat. aq $\mathrm{NaHCO}_{3}(1 \mathrm{~mL})$ and the mixture was extracted with hexanes- $\mathrm{Et}_{2} \mathrm{O}(2: 1,3 \times 1 \mathrm{~mL})$. The organic layers were combined, washed with brine $(3 \times 1 \mathrm{~mL})$, dried $\left(\mathrm{MgSO}_{4}\right)$, and concentrated. Flash chromatography (hexanes-EtOAc, 40:1 $\rightarrow$ 10:1) afforded (+)-artemone (5) as a clear, pale yellow oil; yield: 15.3 $\mathrm{mg}(57 \%)$.

$[\alpha]_{\mathrm{D}}^{23}+49.4\left(\mathrm{c} 0.7, \mathrm{CHCl}_{3}\right) ; R_{\mathrm{f}}=0.82$ (hexanes-EtOAc, 4:1).

IR: $1709,1634 \mathrm{~cm}^{-1}$.

${ }^{1} \mathrm{H}$ NMR (400 MHz, $\mathrm{CDCl}_{3}$ ): $\delta=5.99$ (dd, $J=11,17 \mathrm{~Hz}, 1 \mathrm{H}$ ), 5.90 (dd, $J=11,17 \mathrm{~Hz}, 1 \mathrm{H}$ ), 5.20 (dd, $J=0.9 \mathrm{~Hz}, 17 \mathrm{~Hz}, 1 \mathrm{H}$ ), 5.17 (dd, $J=1.0,11$ $\mathrm{Hz}, 1 \mathrm{H}), 5.15$ (dd, $J=1.6,17 \mathrm{~Hz}, 1 \mathrm{H}), 4.94(\mathrm{dd}, J=1.6,11 \mathrm{~Hz}, 1 \mathrm{H})$, $4.13(\mathrm{dt}, J=5.9,8.7 \mathrm{~Hz}, 1 \mathrm{H}$ ), 3.05 (dq, $J=8.5,6.9 \mathrm{~Hz}, 1 \mathrm{H}), 1.97(\mathrm{~m}, 1$ H), $1.88(\mathrm{~m}, 1 \mathrm{H}), 1.73(\mathrm{~m}, 1 \mathrm{H}), 1.61(\mathrm{~m}, 1 \mathrm{H}), 1.26(\mathrm{~s}, 3 \mathrm{H}), 1.24(\mathrm{~s}, 3$ H), $1.23(\mathrm{~s}, 3 \mathrm{H}), 0.94(\mathrm{~d}, J=6.8 \mathrm{~Hz}, 3 \mathrm{H})$.

${ }^{13} \mathrm{C}$ NMR $\left(100 \mathrm{MHz}, \mathrm{CDCl}_{3}\right): \delta=215.6,144.8,142.3,114.2,111.0,82.6$, $80.8,51.4,46.0,37.5,29.3,26.4,23.2,23.1,15.2$.

\section{Acknowledgment}

This work was supported by a Camille and Henry Dreyfus Foundation Faculty Startup Award, a Henry Dreyfus Teacher-Scholar Award, the Sherman Fairchild Foundation, the Baker Foundation, Merck/AAAS, the Harvey Mudd College Department of Chemistry, and the Christian 
Synthesis

E. D. Nacsa et al.

Scholars Foundation. We thank Dr. Richard W. Kondrat and Ronald B. New at the University of California, Riverside for exact mass measurements, Israel Sanchez for experimental support, and Jason S. Kingsbury for helpful comments on the manuscript.

\section{Supporting Information}

Supporting information for this article is available online at http://dx.doi.org/10.1055/s-0034-1380684.

\section{References}

(1) (a) Naegeli, P.; Klimes, J.; Weber, G. Tetrahedron Lett. 1970, 5021. (b) Misra, L. N.; Chandra, A.; Thakur, R. S. Phytochemistry 1991, 30, 549 .

(2) (a) Vajs, V.; Trifunovic, S.; Janackovic, P.; Sokovic, M.; Milosavljevic, S.; Tesevic, V. J. Serb. Chem. Soc. 2004, 69, 969. (b) Perfumi, M.; Paparelli, F.; Cingolani, M. L. J. Essent. Oil Res. 1995, 7, 387. (c) Schmidt, E.; Bail, S.; Friedl, S. M.; Jirovetz, L.; Buchbauer, G.; Wanner, J.; Denkova, Z.; Slavchev, A.; Stoyanova, A.; Geissler, M. Nat. Prod. Commun. 2010, 5, 1365.

(3) Siska, P.; Fodran, P.; Szolcsanyi, P. Tetrahedron 2014, 70, 6420.

(4) Honda, Y.; Ori, A.; Tsuchihashi, G. Chem. Lett. 1987, 1259.

(5) Wan, K. K.; Evans-Klock, C. D.; Fielder, B. C.; Vosburg, D. A. Synthesis 2013, 45, 1541.
(6) (a) Akhila, A.; Sharma, P. K.; Thakur, R. S. Tetrahedron Lett. 1986, 27, 5885. (b) Thakur, R. S.; Akhila, A. In Newer Trends in Essential Oils and Flavours: Selected Papers Presented at the International Symposium; Dhar, K. L.; Thappa, R. K.; Agarwal, S. G., Eds.; Tata McGraw-Hill: New Delhi, 1993, 51.

(7) Morrison, K. C.; Litz, J. P.; Scherpelz, K. P.; Dossa, P. D.; Vosburg, D. A. Org. Lett. 2009, 11, 2217.

(8) Naegeli, P.; Weber, G. Tetrahedron Lett. 1970, 959.

(9) Gaich, T.; Baran, P. S. J. Org. Chem. 2010, 75, 4657.

(10) Sharma, M. L.; Chand, T. Proc. Indian Acad. Sci. (Chem. Sci.) 1996, 108, 21.

(11) Melchiorre, P. Angew. Chem. Int. Ed. 2012, 51, 9748.

(12) (a) Marigo, M.; Wabnitz, T. C.; Fielenbach, D.; Jørgensen, K. A. Angew. Chem. Int. Ed. 2005, 44, 794. (b) Hayashi, Y.; Gotoh, H.; Hayashi, T.; Shoji, M. Angew. Chem. Int. Ed. 2005, 44, 4212. (c) Ishikawa, H.; Suzuki, T.; Hayashi, Y. Angew. Chem. Int. Ed. 2009, 48, 1304. (d) Ishikawa, H.; Honma, M.; Hayashi, Y. Angew. Chem. Int. Ed. 2011, 50, 2824.

(13) Fleury, L. M.; Ashfeld, B. L. Org. Lett. 2009, 11, 5670.

(14) (a) Richter, J. M.; Ishihara, Y.; Masuda, T.; Whitefield, B. W.; Llamas, T.; Pohjakallio, A.; Baran, P. S. J. Am. Chem. Soc. 2008, 130, 17938. (b) Burns, N. Z.; Baran, P. S.; Hoffmann, R. W. Angew. Chem. Int. Ed. 2009, 48, 2854.

(15) Sabitha, G.; Prasad, M. N.; Bhikshapathi, M.; Yadav, J. S. Synthesis 2010, 807. 